\title{
Wind farm power production in the changing wind: Robustness quantification and
} layout optimization

\author{
Feng, Ju; Shen, Wen Zhong
}

Published in:

Energy Conversion and Management

Link to article, DOI:

10.1016/j.enconman.2017.06.005

Publication date:

2017

Document Version

Peer reviewed version

Link back to DTU Orbit

\section{Citation (APA):}

Feng, J., \& Shen, W. Z. (2017). Wind farm power production in the changing wind: Robustness quantification and layout optimization. Energy Conversion and Management, 148, 905-914.

https://doi.org/10.1016/j.enconman.2017.06.005

\section{General rights}

Copyright and moral rights for the publications made accessible in the public portal are retained by the authors and/or other copyright owners and it is a condition of accessing publications that users recognise and abide by the legal requirements associated with these rights.

- Users may download and print one copy of any publication from the public portal for the purpose of private study or research.

- You may not further distribute the material or use it for any profit-making activity or commercial gain

- You may freely distribute the URL identifying the publication in the public portal 


\title{
Wind farm power production in the changing wind: robustness
}

\section{quantification and layout optimization}

\author{
Ju Feng*, Wen Zhong Shen
}

Department of Wind Energy, Technical University of Denmark, DK-2800 Lyngby, Denmark

\section{Abstract}

Wind farms operate often in the changing wind. The wind condition variations in a wide range of time scales lead to the variability of wind farms' power production. This imposes a major challenge to the power system operators who are facing a higher and higher penetration level of wind power. Thus, wind farm developers/owners need to take the variability into consideration in the designing/planning stage, in addition to the conventional main objective of maximizing the expected power output under a fixed wind distribution. In this study, we first propose a new metric to evaluate the variability of wind power based on the characteristics of the wind farm and its local wind conditions. Then a series of robustness metrics are proposed to quantify wind farm's ability to produce power with high mean value and low variability under changing wind, considering both short-term and long-term wind condition variations. Based on these metrics, wind farm layout optimization is performed to maximize the robustness of a real offshore wind farm in Denmark. The results demonstrate that the robustness metrics are more flexible and complete than the conventional metrics for characterizing wind farm power production, such as mean power output or wind power variability alone, and it is feasible to design wind farms to produce power with high mean value and low variability.

Keywords: wind power; wind farm; wind variation; variability; robustness quantification; layout optimization

${ }^{*}$ Corresponding author. Tel.: +45 45254334;

E-mail address: jufen@dtu.dk (J. Feng). 


\begin{tabular}{|c|c|}
\hline Acronyms & \\
\hline WF & wind farm \\
\hline WT & wind turbine \\
\hline AEP & annual energy production \\
\hline TRI & terrain ruggedness index \\
\hline Symbols & \\
\hline$v$ & wind speed $[\mathrm{m} / \mathrm{s}]$ \\
\hline$\theta$ & wind direction $[\mathrm{deg}]$ \\
\hline$P$ & power output [MW] \\
\hline $\bar{v}, \bar{\theta}, \bar{P}$ & non-dimensionalized wind speed, wind direction and power [-] \\
\hline$A_{k}$ & scale factor of Weibull distribution for the $k$ th sector $[\mathrm{m} / \mathrm{s}]$ \\
\hline$c_{k}$ & shape factor of Weibull distribution for the $k$ th sector [-] \\
\hline$f_{k}$ & frequency of occurrence for the $k$ th sector [-] \\
\hline$\theta_{k}$ & wind direction for the center of the $k$ th sector [deg] \\
\hline$P_{\text {mean }}$ & mean power output of the WF [MW] \\
\hline$P_{\text {rated }}$ & nameplate capacity of the WF [MW] \\
\hline$v_{\text {in }}, v_{\text {out }}$ & minimal and maximal wind speeds the WF produces power $[\mathrm{m} / \mathrm{s}]$ \\
\hline PSRI & power surface ruggedness index [-] \\
\hline VoP & variability of power [-] \\
\hline$P_{\text {mean }}^{r e f}$ & mean power output of the reference WF $[\mathrm{MW}]$ \\
\hline VoP $P^{r e f}$ & variability of power of the reference WF [-] \\
\hline$\tilde{A}_{k}, \tilde{c}_{k}, \tilde{f}_{k}$ & variated version of $A_{k}, c_{k}$ and $f_{k}$ to account for long term wind variations \\
\hline$\xi_{k}^{A}, \xi_{k}^{c}, \xi_{k}^{f}$ & independent random variables to characterize the parametric variations of $A_{k}, c_{k}$ and $f_{k}$ \\
\hline$V R_{A}, V R_{c}, V R_{f}$ & maximal variation percentages of $A_{k}, c_{k}$ and $f_{k}[-]$ \\
\hline$p_{\lambda}$ & probability density function of the $\lambda$-PDF distribution [-] \\
\hline
\end{tabular}




\begin{tabular}{|ll|}
\hline$\lambda$ & shape parameter of the $\lambda$-PDF distribution [-] \\
$a_{\lambda}$ & normalizing parameter of the $\lambda$-PDF distribution [-] \\
$N_{\text {sample }}$ & number of variated wind condition samples [-] \\
$P_{\text {mean }}^{l}$ & mean power output of the WF under the $l$ th variated wind condition sample $[\mathrm{MW}]$ \\
$R_{\text {short }}, R_{\text {long }}, R_{\text {overall }}$ & short-term, long-term and overall robustness metrics [-] \\
$\alpha, \beta, \gamma$ & weighting parameters in $R_{\text {short }}, R_{\text {long }}$ and $R_{\text {overall }}[-]$ \\
$N_{w t}$ & number of WTs in the WF [-] \\
$\boldsymbol{X}, \boldsymbol{Y}$ & vectors of $x$ and $y$ coordinates describing all WTs' locations $[\mathrm{m}]$ \\
\hline
\end{tabular}

\section{Introduction}

In the past two decades, wind energy has grown into a mature and important player in the global energy mixture, especially in the countries where environmental concerns and sustainability have received a high priority in their development goals. For example, in Denmark, one of the pioneering countries supporting wind power development, the penetration of wind energy production in the total electricity consumption has grown from $17 \%$ in 2006 to $42 \%$ in 2015 [1]. With the higher and higher penetration level of wind power, the integration challenges faced by the electrical power system are becoming more and more critical.

From the perspective of power system operators, the power variability is one of the most crucial challenges brought by the high wind power penetration. The wind power variability is a direct consequence of the fluctuating nature of wind and makes the wind power difficult or generally impossible to dispatch like other conventional powers. This imposes negative impacts on the reliability, stability, operations, ancillary services and cost of the power system [2].

The variability of wind power has been the subject of many studies. Most of these studies focus on the shortterm variability and are mainly based on the study of the wind power time-series. For example, Katzenstein et al. [3] developed a metric to quantify the sub-hourly variability cost of individual wind farms (WFs). Kivilouma et al. [4] studied the characteristics of wind power variability using real data from multiple regions and divided these regions into low, medium and high variability regions according to the maximum 1 hour wind power ramps relative to the nominal capacity. The metric they used to quantify the short-term variability consists of 
values of the ramp duration and the exceedance level of the ramp magnitude. Boutsika et al. [5] proposed a conditional range metric to quantify the intra-hour wind power variability and extended it to consider scarce or noisy data situations in a recent study [6]. Power spectral density has also been applied in other variability metrics $[7,8]$.

Several methods have also been proposed to mitigate the negative impacts brought by the short-term variability of wind power, including: interconnecting WFs [8], optimizing regional spatial distribution of WFs [9] and optimizing wind turbine (WT) control strategy [10]. Better wind power forecasting can also contribute in solving the short-term variability challenges, as suggested in several recent studies, such as in $[11,12]$.

In the meanwhile, the long-term variability of wind power has received less attention, mainly due to the lack of wind power time series that is long enough for long-term variability analysis. Recently, Kirchner-Bossi et al. [13] analyzed the long-term variability of wind power output from a real WT in the period 1871-2009 for two locations in Spain. They found that the simulated annual power output from a WT variates largely from year to year. For example, in one of the sites, its maximal annual value was $644 \mathrm{~kW}$ in 1978 while the minimal value was $485 \mathrm{~kW}$ in 1911. In general, predicting the long-term variation of wind power is extremely challenging, as there are no accurate methods to predict the long-term variation of wind conditions. As pointed out by Watson [14], general circulation models can predict possible future decadal fluctuations of wind conditions, but large uncertainties still exist.

All the studies referred above focused on the variability of wind power, which is of major concern to the power system operators. However, from the perspective of WF developers/owners, the annual energy production (AEP) of a WF is far more important than its variability, since it directly determines the WF's income. Thus, maximizing AEP is usually one of the most important objectives for any WF designer [15].

In the designing/planning stage, AEP of a WF is calculated as an expected value based on the local wind distribution, which is derived from a certain period of wind measurement data [16]. Most of the studies on WF layout optimization assumed a fixed wind distribution and thus didn't take the long-term variability into consideration [15]. Although the short-term variability has been considered for WF operation and control [10], it is seldom considered in the designing/planning stage. One exception is a recent study by Song et al. [17]. In this study, they defined a sensitivity index to evaluate the variation of a given WF's power output under varying wind directions. After a first stage optimization of maximizing the mean power output, they then carried out a 
second stage local adjustment of the layout to minimize the sensitivity of power to the changing wind direction.

Ideally, it is desirable to have the mean value of WF's power output as high as possible while keeping its variability under both short-term and long-term wind variations as low as possible. A lot of studies in the literature have focused on maximizing the power output, through both wind farm layout optimization $[18,19]$ and wind farm control $[20,21]$. However, to the authors' knowledge, there hasn't been a metric in the literature that can be used to address both aspects in the same time, i.e., maximizing the mean power output and minimizing its variability.

In this study, we propose a series of robustness metrics to quantify WF's ability to produce power with high mean value and low variability under changing wind. A new metric for the variability of power is first proposed to quantify the sensitivity of a given WF's power output to the possible short-term wind condition variations, weighted by the local joint distribution of wind speed and wind direction. Then, the short-term robustness is defined by WF's mean power output and its variability of power. The long-term robustness is computed by modelling the possible long-term wind condition variations and investigating WF's power outputs under such variating/fluctuating wind conditions. Finally, the overall robustness is defined as a weighted sum of these two robustness metrics and a layout optimization study is then carried out to maximize it for the Horns Rev 1 WF using the random search algorithm [19].

\section{Wind farm power output}

A WF is a group of WTs located at a site to generate power from the wind. It can be viewed as a system that transforms the wind energy into power. At any given moment, the power output of a WF depends mainly on the characteristics of the inflow wind and the state of the WF itself.

The most essential characteristics of the inflow wind are wind speed $v$ and wind direction $\theta$ at hub height. Although other characteristics such as turbulence intensity, atmospheric stability also have impacts on the power output, their influence on WF's long-term mean performance can be neglected.

In order to calculate the mean power output of a WT or a WF, we usually model the wind with a certain probability distribution for wind speed. While different types of distributions are available [22], the most widely used is the Weibull distribution, combined with a wind rose that describes the frequencies of occurrence of different directional sectors [16]. Considering the fact that wind speed and wind direction are not independent 
random variables, we can better characterize the wind conditions with a joint distribution of wind speed and wind direction: $P d f=P d f(v, \theta)$. Such kind of joint distribution can be obtained based on wind measurement data with the method proposed in Ref. [23]. Parameters used in this method include the sector wise Weibull distribution parameters (scale factor $A_{k}$ and shape factor $\left.c_{k}\right)$ and the related relative frequency of occurrence $\left(f_{k}\right)$ for each sector that is centered at direction $\theta_{k}$. The number of sectors is commonly set as 12 , i.e., with $k=$ $1, \ldots, 12$.

Note that $\theta_{k}$ is usually fixed as $\theta_{k}=(k-1) * 30 \mathrm{deg}$, and the relative frequency of occurrence $f_{k}$ must satisfy the condition $\sum f_{k}=1$. So we can determine a joint distribution $P d f=P d f(v, \theta)$ with 35 independent parameters, if the common practice of using 12 directional sectors is chosen.

For the state of WF, the most important factors for power output are the characteristics of WTs, the layout of $\mathrm{WF}$, the terrain features of the WF site, and the operational status of the WF. When investigating the mean power production of a WF in the designing/planning stage, we can reasonably assume its operation status to be perfect, i.e., all WTs are fully operational and no curtailments are required.

For such a WF, we can then calculate its total power output as a function of the inflow wind speed $v$ and wind direction $\theta$, i.e., $P=P(v, \theta)$. This can be done by: discretizing the interested wind speed range and wind direction range into $\left[v_{i}\right]$ and $\left[\theta_{j}\right]$; simulating the WF flow field for each pair of $\left(v_{i}, \theta_{j}\right)$ using a certain wake model such as the Jensen wake model; and calculating the power outputs of each WT and the whole WF accordingly. The detailed procedure of doing this calculation can be found in Ref. [19]. In Ref. [23], the resolution for the discretization is recommended to be $\Delta v=1 \mathrm{~m} / \mathrm{s}$ and $\Delta \theta=1 \mathrm{deg}$, which is suggested for reliable WF layout optimization studies. Thus we use this set of resolution in our study.

As we know, the power output of a single WT depends only on the wind speed $v$ and the function $P=P(v)$ which is called the power curve. Similarly, the power output of a WF depends on both the wind speed $v$ and the wind direction $\theta$. Thus, the function $P=P(\nu, \theta)$ can be viewed as an extension of the power curve and called as the power surface. Like the power curve, the power surface of a WF describes its power production characteristics and is determined solely by its own state.

As an example, the power surface of the Horns Rev 1 offshore WF and its local wind distribution are shown in Figure 1. This WF is an offshore WF in Denmark composed of 80 Vestas 2MW WTs, and it has been used as test cases in Refs. [19,23]. 

output for this WF as:

$$
P_{\text {mean }}=\iint P(v, \theta) P d f(v, \theta) d v d \theta
$$

Assuming the availability factor is $100 \%$, we can calculate this WF's annual energy production (AEP), which measures the amount of energy it produces in an average year, as:

$$
A E P=8770 \times P_{\text {mean }}
$$

where 8770 is the total number of hours in a year.

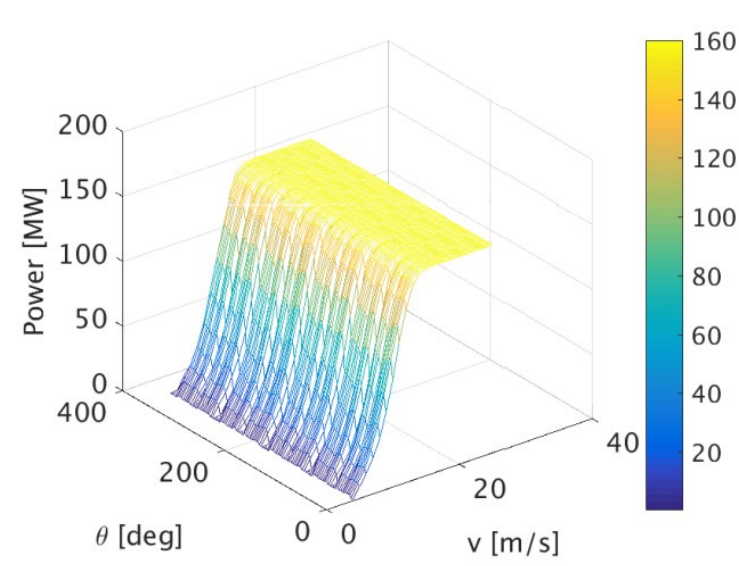

(a)

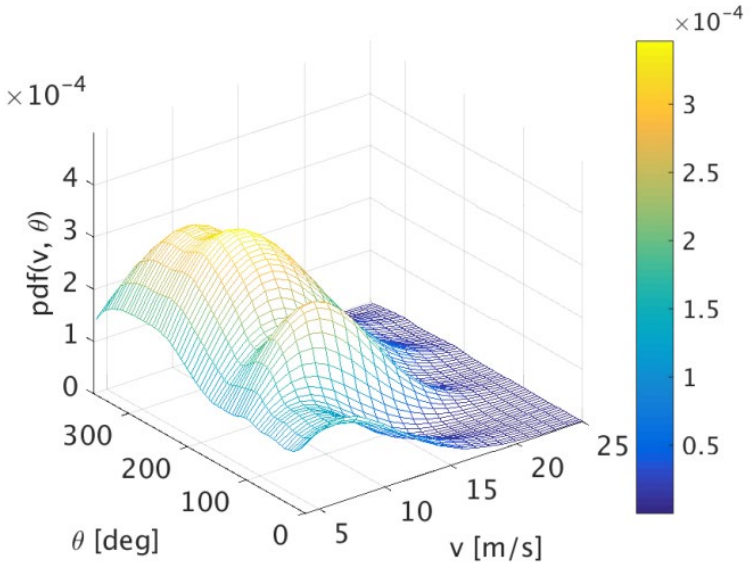

(b)

Figure 1. (a) power surface and (b) local wind distribution of the Horns Rev 1 wind farm.

\section{Variability of power output}

Wind power variates at a wide range of time scales. In order to take the consideration of variability into the design of WFs, we propose a new metric to quantify the variability of a given WF's power output against the short-term wind condition change.

Most studies on wind power variability tackle the problem by studying the wind power time series. While it is a natural choice to analyze real wind power output time series, it can be difficult to implement in the WF designing/planning stage. Since at this stage, we typically have not finalized the WF design yet and also face a lack of information about the time series of wind speed and wind direction that this WF will face in the future. Although we usually have access to wind measurement data for the past 2-3 years, from which we can obtain 
historical time series of wind speed and wind direction, and also the local wind distribution, we can't assume the historical time series of wind conditions are very representative of the real time series of wind conditions that this WF will face in the future.

However, we can be more confident to assume that the joint distribution of wind speed and wind direction is more representative of the future wind distribution, since it is a statistical measure based on several years measurements. Furthermore, it is also the common practice to make a projection of the AEP of the designed WF in its lifetime based on this distribution. Thus, we can define our metric of wind power variability on the local wind distribution instead of some historical or artificial wind condition time series.

In principle, the variability of WF's power output can be viewed as the consequence of the variability of wind conditions it faces and multiplied by the sensitivity of its power output to wind condition variations. Higher wind variability and/or higher sensitivity lead to higher power variability.

The sensitivity of WF's power output against the wind condition variations is determined by WF's state, which mainly includes the WT characteristics, the layout, the terrain feature and the operation status. For a given WF in perfect operation status, this sensitivity can be characterized by its power surface.

Considering a conventional power plant, which can output the rated power regardless of the wind condition, we can draw its power surface in a similar manner as the one for a WF. Since the conventional power plant's power output doesn't depend on the wind conditions, its power surface will be a horizontal plain that is perfectly flat and its sensitivity to wind variations will be zero.

We can view the power surface, whether it is for a WF or for a conventional power plant, as a 'terrain map', where power output defines the 'elevation', wind speed represents the 'x coordinate' and wind direction is the ' $y$ coordinate'. Then we can define an index to quantify the ruggedness of the power surface in a similar way as the terrain ruggedness is quantified. This ruggedness index of the power surface is then used as a measure of the sensitivity to wind condition variations of WF's or the conventional power plant's power output, since it quantifies how much the power output changes when the wind conditions change.

We adopt the calculation method of terrain ruggedness index (TRI) proposed by Riley et al. [24] to calculate the power surface ruggedness index $(P S R I)$. TRI is an objective quantitative measure of topographic heterogeneity and can be easily calculated based on digital elevation data. The TRI value of a given grid cell is calculated based on its surrounding eight cells and the cell size. 
In the case of power surface, we first carry out a non-dimensionalization to the involved quantities $P, v$ and $\theta$ with

$$
\bar{P}=\frac{P}{P_{\text {rated }}}, \quad \bar{v}=\frac{v-v_{\text {in }}}{v_{\text {out }}-v_{\text {in }}}, \quad \bar{\theta}=\frac{\theta}{360^{\circ}}
$$

where $P_{\text {rated }}$ is the nameplate capacity of the WF, $v_{\text {in }}$ and $v_{\text {out }}$ denote the cut-in and cut-out wind speeds of the WF, i.e., the minimal wind speed at which this WF starts to produce power, and the maximal wind speed at which it continues to produce power. For the WFs in flat terrain or offshore, they are the same as the cut-in and cut-out wind speeds of the WTs. Noticing that the maximal power that a WF can output is its nameplate capacity and the interested wind speed at which the WF produces power lies in the range of $\left[v_{\text {in }}, v_{\text {out }}\right]$, we can see that the dimensionless quantities $\bar{P}, \bar{v}$ and $\bar{\theta}$ are all in the range of $[0,1]$. For the joint distribution of the dimensionless wind speed and wind direction, we can easily derive as:

$$
\overline{P d f}(\bar{v}, \bar{\theta})=\operatorname{Pdf}(v, \theta)
$$

where $v=v_{\text {in }}+\left(v_{\text {out }}-v_{\text {in }}\right) \times \bar{v}$ and $\theta=360^{\circ} \times \bar{\theta}$.

For the dimensionless power surface governed by $\bar{P}=\bar{P}(\bar{v}, \bar{\theta})$, let's consider a grid cell of wind condition $(\bar{v}, \bar{\theta})$ and its surrounding cells that have slightly different wind conditions. Assuming the cell size is $\Delta \bar{v}$ by $\Delta \bar{\theta}$, we can plot the central cell with its eight surrounding cells as in Figure 2.

\begin{tabular}{|c|c|c|}
\hline $\begin{array}{c}\bar{P}_{N W}=\bar{P}(\bar{v}-\Delta \bar{v}, \bar{\theta}+\Delta \bar{\theta}) \\
(\bar{v}-\Delta \bar{v}, \bar{\theta}+\Delta \bar{\theta})\end{array}$ & $\begin{array}{c}\bar{P}_{N}=\bar{P}(\bar{v}, \bar{\theta}+\Delta \bar{\theta}) \\
(\bar{v}, \bar{\theta}+\Delta \bar{\theta})\end{array}$ & $\begin{array}{c}\bar{P}_{N E}=\bar{P}(\bar{v}+\Delta \bar{v}, \bar{\theta}+\Delta \bar{\theta}) \\
(\bar{v}+\Delta \bar{v}, \bar{\theta}+\Delta \bar{\theta})\end{array}$ \\
\hline $\bar{P}_{W}=\bar{P}(\bar{v}-\Delta \bar{v}, \bar{\theta})$ & $\bar{P}_{C}=\bar{P}(\bar{v}, \bar{\theta})$ & $\bar{P}_{E}=\bar{P}(\bar{v}+\Delta \bar{v}, \bar{\theta})$ \\
$(\bar{v}-\Delta \bar{v}, \bar{\theta})$ & $(\bar{v}, \bar{\theta})$ & $(\bar{v}+\Delta \bar{v}, \bar{\theta})$ \\
\hline $\begin{array}{c}\bar{P}_{S W}=\bar{P}(\bar{v}-\Delta \bar{v}, \bar{\theta}-\Delta \bar{\theta}) \\
(\bar{v}-\Delta \bar{v}, \bar{\theta}-\Delta \bar{\theta})\end{array}$ & $\bar{P}_{S}=\bar{P}(\bar{v}, \bar{\theta}-\Delta \bar{\theta})$ & $\bar{P}_{S E}=\bar{P}(\bar{v}+\Delta \bar{v}, \bar{\theta}-\Delta \bar{\theta})$ \\
$(\bar{v}, \bar{\theta}-\Delta \bar{\theta})$ & $(\bar{v}+\Delta \bar{v}, \bar{\theta}-\Delta \bar{\theta})$ \\
\hline
\end{tabular}

Figure 2. Grid cell of wind condition $(\bar{v}, \bar{\theta})$ and its surrounding cells.

Then this cell's PSRI can be calculated as: 


$$
\begin{aligned}
\operatorname{PSRI}(\bar{v}, \bar{\theta})=\{ & \left\{\frac{\left(\bar{P}_{C}-\bar{P}_{W}\right)^{2}+\left(\bar{P}_{C}-\bar{P}_{E}\right)^{2}}{\Delta \bar{v}^{2}}+\frac{\left(\bar{P}_{C}-\bar{P}_{N}\right)^{2}+\left(\bar{P}_{C}-\bar{P}_{S}\right)^{2}}{\Delta \bar{\theta}^{2}}\right. \\
& \left.\left.+\frac{\left(\bar{P}_{C}-\bar{P}_{N W}\right)^{2}+\left(\bar{P}_{C}-\bar{P}_{S W}\right)^{2}+\left(\bar{P}_{C}-\bar{P}_{N E}\right)^{2}+\left(\bar{P}_{C}-\bar{P}_{S E}\right)^{2}}{\Delta \bar{v}^{2}+\Delta \bar{\theta}^{2}}\right] / 8\right\}^{1 / 2}
\end{aligned}
$$

Note that in the above formula, $\Delta \bar{v}$ and $\Delta \bar{\theta}$ are chosen corresponding to the recommended cell sizes by Ref. [23]: $\Delta v=1 \mathrm{~m} / \mathrm{s}$ and $\Delta \theta=1 \mathrm{deg}$. Applying Equation (5) to the power surface of a conventional plant, we will obtain zero for any wind conditions due to the perfect flatness of the surface. In contrary, PSRI calculated for the WF will be different for different wind conditions. PSRI of a given wind condition $(\bar{v}, \bar{\theta})$ measures how much the power output will change when the wind condition slightly changes. Thus, it can be viewed as the sensitivity of power output against the possible short-term wind variations from this given wind condition.

However, when designing a WF, we are not only interested in the power variability for any given wind condition. Instead, we need to know the overall variability of power output against all possible wind condition variations. Considering that the joint distribution of wind speed and wind direction $\overline{P d f}(\bar{v}, \bar{\theta})$ quantifies the probability of the wind condition $(\bar{v}, \bar{\theta})$, we can define a new metric called variability of power $(V o P)$ to quantify the overall variability of power output against the short-term wind variations, which is governed by:

$$
V o P=\iint \operatorname{PSRI}(\bar{v}, \bar{\theta}) \overline{P d f}(\bar{v}, \bar{\theta}) d \bar{v} d \bar{\theta}
$$

To test this new metric, we carry out a layout optimization study of the Horns Rev 1 WF to minimize its VoP using the random search algorithm [19], which is briefly described in Section 5. The results are shown in Figure 3. 


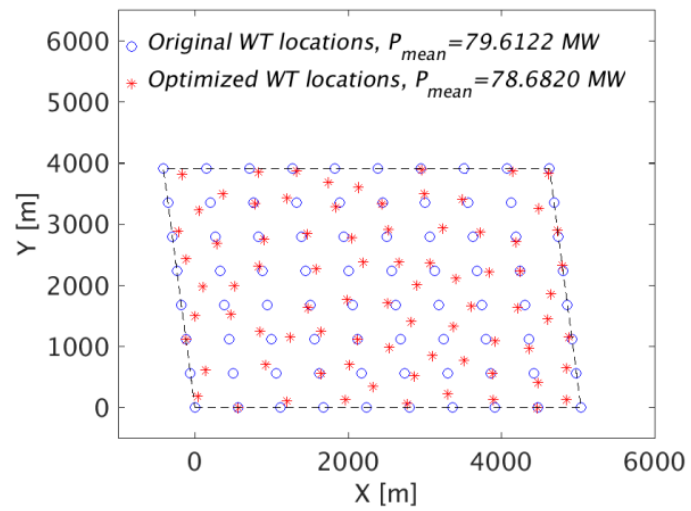

(a)

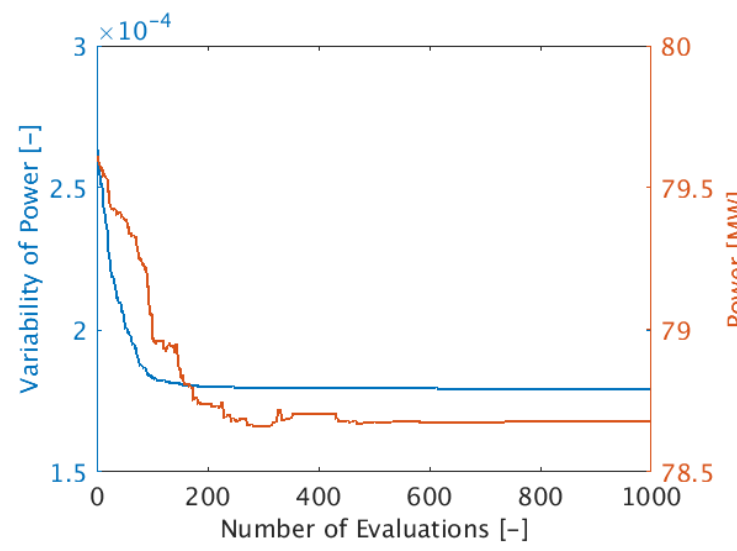

(c)

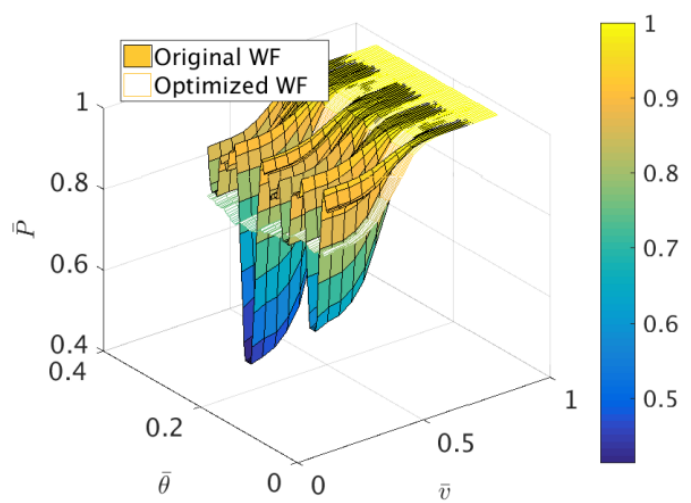

(b)

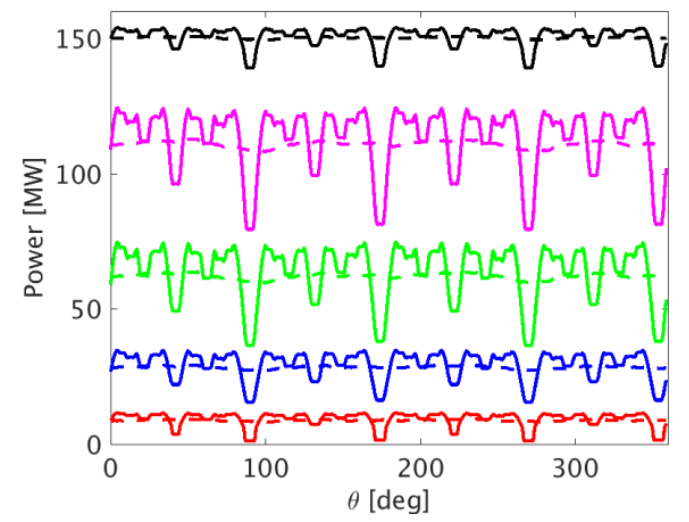

(d)

Figure 3. Layout optimization results of Horns Rev 1 WF to minimize VoP: (a) layouts; (b) partial dimensionless power surfaces; (c) evolution histories of $V o P$ and $P_{\text {mean }}$ in the optimization process; (d) power outputs for all wind directions with different wind speeds (from bottom to top: $v=5,7,9,11,13 \mathrm{~m} / \mathrm{s}$ ) of the original (solid lines) and optimized (dash lines) WFs

Figure 3(a) presents the original and optimized layouts, with their mean power output marked. Note that the mean power has been decreased from 79.61MW to 78.68MW. Figure 3(b) shows parts of the dimensionless power surfaces for the original and optimized WFs. Comparing these two surfaces, we can clearly see that the dimensionless power surface has been largely flattened after optimization, which signifies the sensitivity of the WF's power output against short term wind condition variations has been decreased. The evolutions of $V o P$ and $P_{\text {mean }}$ in the optimization process are shown in Figure 3(c). It shows that $V o P$ has been reduced from 2.63e-4 to 1.80e-4. To better visualize the sensitivity reduction, Figure 3(d) shows the power output of the original and 
optimized WFs for all wind directions under a range of different wind speeds. The comparison shows that the sensitivity of the WF's power output against wind direction changes has been significantly reduced.

To further validate the effectiveness of the proposed variability metric, we also compare these two WF layouts using the conventional wind power variability metric. This metric is based on the statistical analysis of the wind power time series and defined as the standard deviation (std) of the corresponding power ramps, i.e., stepchanges of power output taken over a given time frame [5].

Using the 3 year time series of wind speed and wind direction measured at Horn Rev [23], we can easily simulate the corresponding power output time series from the original and optimized WFs based on their power surfaces. Then we can calculate the standard deviations of $10 \mathrm{~min}$ power ramps as $11.22 \mathrm{MW}$ for the original WF and 10.13MW for the optimized WF. Thus, the standard deviation of $10 \mathrm{~min}$ power ramps has been reduced $9.7 \%$ after the optimization. To better illustrate the difference between these two sets of power time series, we randomly choose 10 samples of segments in the 3 year time series with different lengths (1 hour, 1 day and 1 week) and calculate the standard deviations related to the $10 \mathrm{~min}$ power ramps. The comparisons between the standard deviations for the 10 samples and the power output time series for 1 sample are shown in Figure 4, in which the power ramp and power have been normalized based on the WF's nameplate capacity.
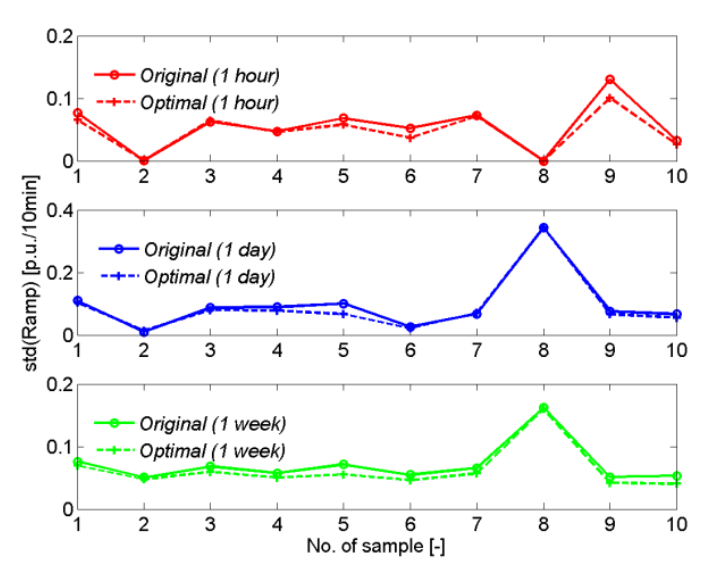

(a)
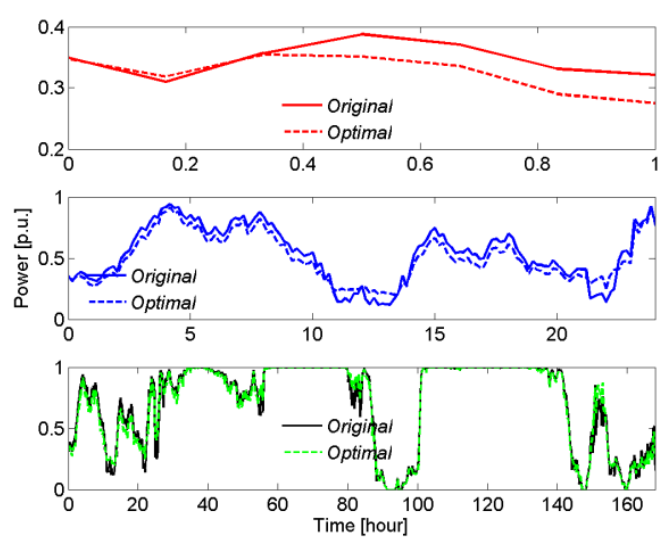

(b)

Figure 4. Characteristics of wind power time series of the original and optimized WF in 3 time intervals: (a) standard deviations of $10 \mathrm{~min}$ power ramp of 10 samples; (b) power output of a sample.

Considering that the standard deviation of $10 \mathrm{~min}$ power ramps measures the short-term variability of the power time series, we can clearly see from the above figure that the wind power time series from the optimized 
WF generally exhibits smaller variability in different time ranges than that from the original WF. This demonstrates that the new metric $V o P$ could characterize the short-term power variability of WFs quite well as the conventional time-series based metrics.

Further examining the optimization results, we can see that the variability of power has been significantly improved as suggested by the large reduction of $V o P$, but it comes with an expensive cost, i.e., the decreased mean power. Since lower mean power means less energy production and thus less income for the WF's owner, we can conclude that the optimization to minimize $V o P$ alone is unacceptable and advanced metrics need to be considered for WF design. Thus, we propose the metrics of robustness in the next section.

\section{Quantification of robustness}

Robustness of WF's power production against the changing wind is defined as a type of metric that quantifies the WF's ability to maintain its power production performance under wind condition variations. Using this type of metric, we can simultaneously address two usually conflicting objectives for a WF, i.e., maximizing the power output and minimizing its variability.

Considering the different time scales of wind variations, we divide the robustness into two parts: short-term robustness and long-term robustness. And then an overall robustness can be defined as a weighted sum of these two parts.

\subsection{Short-term robustness}

We define short-term robustness $R_{\text {short }}$ as the robustness of wind farm power output against short-term wind variations. Note that the variability of power against short-term wind variations can be quantified by the metric proposed in the previous section, $V O P$. For a WF, it is important to reduce its variability of power, and it is even more important to maintain its mean power output at a high level. Thus, we define the short-term robustness of a given WF as:

$$
R_{\text {short }}=\frac{\left(P_{\text {mean }} / P_{\text {mean }}^{\text {ref }}\right)^{\alpha}}{\left(\text { VoP } / \text { VoP } P^{\text {ref }}\right)^{1-\alpha}}, \quad 0 \leq \alpha \leq 1
$$

where $P_{\text {mean }}^{r e f}$ and $V o P^{r e f}$ are the mean power and the power variability of a reference WF, which can be an initial design of this given WF that acts as a baseline design and $\alpha$ is a weighting parameter to address the trade- 
off between the two objectives: maximizing $P_{\text {mean }}$ and minimizing $V o P$. When maximizing $R_{\text {short }}$ of a WF, we can easily adjust our emphasis on these two objectives. For example, maximizing $R_{\text {short }}$ is identical to maximizing $P_{\text {mean }}$ alone if $\alpha=1$ is chosen, while minimizing $\operatorname{VoP}$ alone requires $\alpha=0$.

To select the proper value for $\alpha$, we conduct layout optimizations for the Horns Rev 1 WF to maximize its $R_{\text {short }}$ with a range of different values for $\alpha(\alpha=0,0.05,0.5,0.95,1)$. Each case is optimized with 10000 evaluations. The obtained results are presented in Table 1.

Table 1. Performance summary of the original and optimized WF with different $\alpha$

\begin{tabular}{lll}
\hline & $P_{\text {mean }}[\mathrm{MW}]$ & VoP $[-]$ \\
\hline Original & 79.61 & $2.63 \mathrm{e}-4$ \\
Optimal $(\alpha=0)$ & $78.67(-1.18 \%)$ & $1.79 \mathrm{e}-4(-31.90 \%)$ \\
Optimal $(\alpha=0.05)$ & $78.83(-0.98 \%)$ & $1.79 \mathrm{e}-4(-31.90 \%)$ \\
Optimal $(\alpha=0.5)$ & $79.28(-0.42 \%)$ & $1.80 \mathrm{e}-4(-31.82 \%)$ \\
Optimal $(\alpha=\mathbf{0 . 9 5})$ & $\mathbf{7 9 . 6 4 ( + \mathbf { 0 . 0 4 } \% )}$ & $\mathbf{1 . 8 1 e - 4}(\mathbf{- 3 1 . 4 4 \% )}$ \\
Optimal $(\alpha=1)$ & $79.72(+0.14 \%)$ & $2.10 \mathrm{e}-4(-20.27 \%)$ \\
\hline
\end{tabular}

Note that in the above table, also in Table 2 and 3 in this paper, percentages in the brackets represent the relative differences compared with the original WF. After examining the results, we select $\alpha=0.95$, since it gives most balanced results on both objectives: increasing $P_{\text {mean }}$ and decreasing $V o P$.

\subsection{Long-term robustness}

Similarly, we can consider the impact of long term wind variations on the WF power production. It is well known that the wind condition variates in the long term, which causes the long term variability of power production of WFs [25]. However, there is no adequate method to predict the long-term variations of wind conditions accurately. Although measure-correlate-predict (MCP) methods can help estimating the average long term wind condition [26,27], it can't predict the long term wind variations such as inter-annual changes of wind distribution.

In order to account the long term variability of wind conditions in WF design, we assume: 
- the joint distribution $\operatorname{Pd} f(v, \theta)$ derived from the wind measurement data represents the mean wind condition in WF's lifetime;

- the uncertainty caused by the long term wind condition variations can be modelled by adding random variations to the joint distribution's parameters;

- the random variations of parameters are bounded and follow certain statistical distributions.

Based on these assumptions, we can then conduct a Monte Carlo simulation of the long term wind variations and assess a given WF's sensitivity to them.

Noting that a joint distribution $\operatorname{Pd} f(v, \theta)$ is usually determined by 35 independent parameters, we can obtain a set of randomly fluctuating parameters as:

$$
\begin{aligned}
& \tilde{A}_{k}=A_{k}+A_{k} \cdot V R_{A} \cdot \xi_{k}^{A}, \\
& \tilde{c}_{k}=c_{k}+c_{k} \cdot V R_{c} \cdot \xi_{k}^{c}, \quad k=1, \ldots, 12 \\
& \tilde{f}_{k}=f_{k}+f_{k} \cdot V R_{f} \cdot \xi_{k}^{f},
\end{aligned}
$$

292

where $\xi_{k}^{A}, \xi_{k}^{c}$ and $\xi_{k}^{f}$ are independent random variables bounded by $[-1,1]$ and follow a given type of distributions; $V R_{A}, V R_{C}$ and $V R_{f}$ denote the maximal variation percentages on the three different parameters; and $\tilde{f}_{k}$ needs to be further normalized to $\tilde{f}_{k}=\tilde{f}_{k} / \sum \tilde{f}_{k}$ since it should satisfy $\sum \tilde{f}_{k}=1$.

In this study we choose the so-called $\lambda$-PDF distribution to model the random parametric variations. This distribution was first introduced by Fang et al. [28] to model a family of bounded, mono-peak, and symmetrical distributions. Later it was used to model bounded uncertain parameters in a study of robustness of feedback stabilization control [29]. Here we assume $\xi_{k}^{A}, \xi_{k}^{c}$ and $\xi_{k}^{f}$ in Equation (7) all follow the $\lambda$-PDF distribution as defined as follows:

$$
p_{\lambda}(\xi)=\left\{\begin{array}{cc}
a_{\lambda} \cdot\left(1-\xi^{2}\right)^{\lambda-0.5}, & |\xi| \leq 1 \\
0, & |\xi|>1
\end{array}\right.
$$

where $\lambda \geq 0$ is a parameter controlling the shape of the distribution, and $a_{\lambda}$ is a normalizing coefficient written as:

$$
a_{\lambda}=\frac{\Gamma(\lambda+1)}{\Gamma(0.5) \Gamma(\lambda+0.5)}
$$


302

in which $\Gamma(\cdot)$ is Gamma function. This distribution can approximate any mono-peak and symmetrical PDFs distributed within $[-1,1]$, as long as they are continuous and smooth [29]. Figure 5 shows the $\lambda$-PDF distributions with different values of $\lambda$.

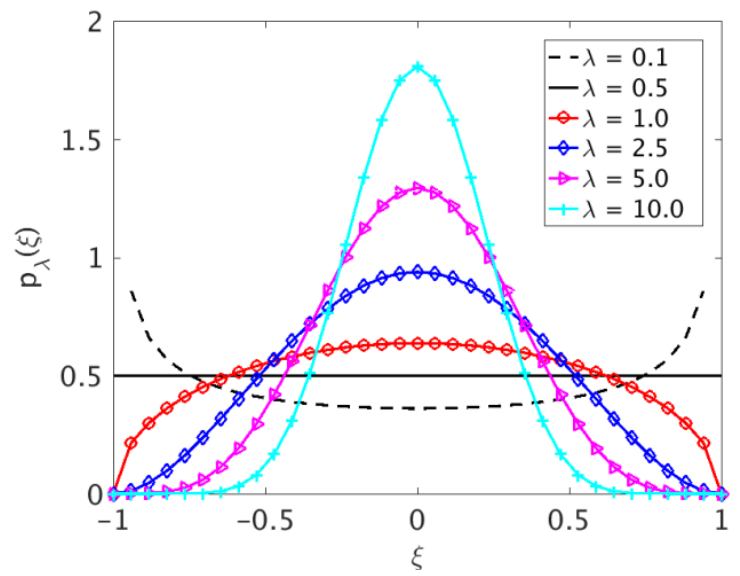

Figure 5. $\lambda$-PDF distributions with different values of $\lambda$.

In this study we use $\lambda$-PDF distributions with $\lambda=1.0$ for all parameters and choose $V R_{A}=0.2, V R_{c}=0.1$ and $V R_{f}=0.5$ for the maximal parametric variation ranges. Employing Equation (7) we can then generate $N_{\text {sample }}$ sets of parameters, each defining a different joint distribution $P d f_{l}(v, \theta)$. We choose $N_{\text {sample }}=1000$ and two of the generated distributions are shown in the following figure.
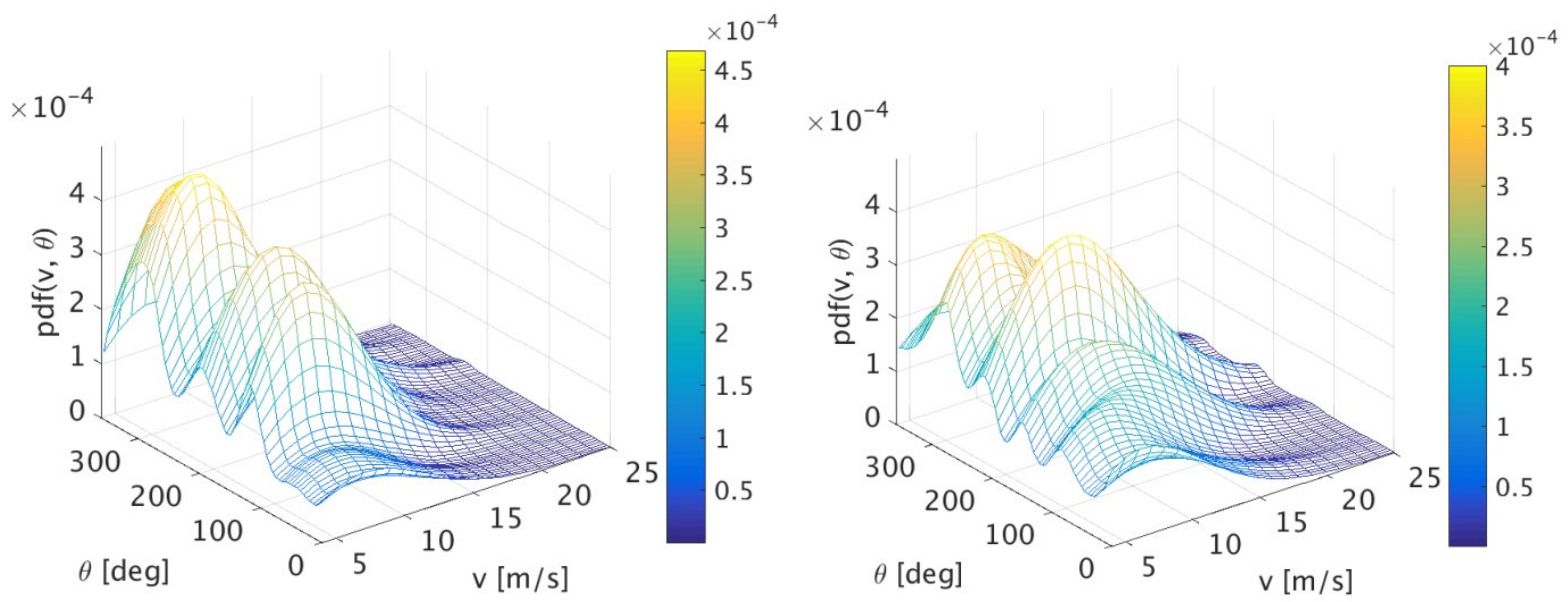

Figure 6. Two wind distributions generated by variated parameters. 
For any given WF, we can then calculate a series of $P_{\text {mean }}$ values: $\left[P_{\text {mean }}^{l}\right]$, each of which represents a possible $P_{\text {mean }}$ that this WF can yield when facing a variated wind distribution characterized by $P d f_{l}(v, \theta)$. Based on the mean and standard deviation of these mean power values, we can define a measure $R_{\text {long }}$ to quantify the longterm robustness, which represents how robust the wind farm is against long-term wind condition variations. It is governed by

$$
R_{\text {long }}=\frac{\left(\operatorname{mean}\left(\left[P_{\text {mean }}^{l}\right]\right)\right)^{\beta}}{\left(\operatorname{std}\left(\left[P_{\text {mean }}^{l}\right]\right)\right)^{1-\beta}}, \quad 0 \leq \beta \leq 1, l=1,2, \ldots, N_{\text {sample }}
$$

In this definition we also address the trade-off between higher expected value of $P_{\text {mean }}$ and lower variations of $P_{\text {mean }}$ under long-term wind condition variations. To determine the optimal weighting parameter $\beta$, a series of layout optimizations are done to maximize $R_{\text {long }}$ of the Horns Rev 1 WF, with results shown in Table 2 .

Table 2. Performance summary of the original and optimized WF with different $\beta$

\begin{tabular}{lcc}
\hline & $\begin{array}{c}\text { mean }\left(\left[P_{\text {mean }}^{l}\right]\right) \\
{[\mathrm{MW}]}\end{array}$ & $\begin{array}{c}\operatorname{std}\left(\left[P_{\text {mean }}^{l}\right]\right) \\
{[\mathrm{MW}]}\end{array}$ \\
\hline Original & 78.54 & 3.482 \\
Optimal $(\beta=0)$ & $78.62(+0.10 \%)$ & $3.479(-0.07 \%)$ \\
Optimal $(\beta=0.05)$ & $78.61(+0.09 \%)$ & $3.479(-0.07 \%)$ \\
Optimal $(\beta=0.5)$ & $78.64(+0.13 \%)$ & $3.480(-0.06 \%)$ \\
Optimal $(\boldsymbol{\beta}=\mathbf{0 . 9 5})$ & $\mathbf{7 8 . 6 5 ( + 0 . 1 4 \% )}$ & $\mathbf{3 . 4 8 0}(\mathbf{- 0 . 0 5 \% )}$ \\
Optimal $(\beta=1)$ & $78.65(+0.15 \%)$ & $3.480(-0.05 \%)$
\end{tabular}

Based on the above results and the need to address both objectives (higher mean value and lower standard deviation), $\beta=0.95$ is chosen for the following optimization studies.

\subsection{Overall robustness}

Finally, the overall robustness can be defined as a weighted combination of the short-term and long-term robustness measures: 


$$
R_{\text {overall }}=\gamma R_{\text {short }}+(1-\gamma) R_{\text {long }}, \quad 0 \leq \gamma \leq 1
$$

where $\gamma$ is a weighting parameter to adjust the trade-off between the short-term and long-term robustness measures.

When maximizing $R_{\text {overall }}$ of a WF, we can easily adjust the emphasis on either the short term or the long term robustness. For example, maximizing $R_{\text {overall }}$ is identical to maximizing $R_{\text {short }}$ alone if $\gamma=1$ is chosen, while choosing $\gamma=0$ is the same as maximizing $R_{\text {long }}$ alone. The choice of an optimal $\gamma$ is discussed in the layout optimization study in next section.

\section{Layout optimization}

Considering a WF composed of $N_{w t}$ WTs, we can represent its layout by $\boldsymbol{X}=\left[x_{1}, x_{2}, \ldots, x_{N_{w t}}\right]$ and $\boldsymbol{Y}=$ $\left[y_{1}, y_{2}, \ldots, y_{N_{w t}}\right]$. Here we assume the WF is composed of a single type of WT. If the type of WTs and the wind conditions at the WF site are given, the WF's overall robustness is a function of its layout. Thus, we can formulate the layout optimization of maximizing the overall robustness as:

$$
\max R_{\text {overall }}=R_{\text {overall }}(\boldsymbol{X}, \boldsymbol{Y})
$$

As Equations $(6,10,11)$ show, there are 3 weighting parameters $(\alpha, \beta$ and $\gamma)$ in the robustness metrics, which we can tune to adjust the focus of the optimization objective. For example, we can adjust the parameters to: maximize $P_{\text {mean }}(\alpha=1, \gamma=1)$; minimize $\operatorname{VoP}(\alpha=0, \gamma=1)$; maximize mean $(\beta=1, \gamma=0)$ and minimize standard deviation $(\beta=0, \gamma=0)$ of $\left[P_{\text {mean }}^{l}\right]$ when facing long-term wind condition variations. For the case study presented here, we use the fixed values for $\alpha$ and $\beta(\alpha=0.95, \beta=0.95)$, combined with a range of different values for $\gamma(\gamma=0,0.05,0.5,0.95,1)$.

Two types of constraints, WF boundary and wind turbine proximity, are also considered in the formulated optimization problem. The detailed modeling of these constraints are the same as in [19] and the minimal distance between any two turbines are set as 5 rotor diameters. The applied optimization method is the random search algorithm as described in [19].

This algorithm is a single solution search method. It keeps the current best layout that has been found and generates a new feasible layout at each step, by randomly choosing one WT, moving it to a random position and 
checking all the constraints. This new layout is then compared with the current best layout. If the new layout is better, it replaces the current best one. This step is iteratively repeated until a stop condition is met. Normally, the stop condition can be set as a given maximal number of steps (or WF evaluations). The procedure of this algorithm is depicted in the following flow chart.

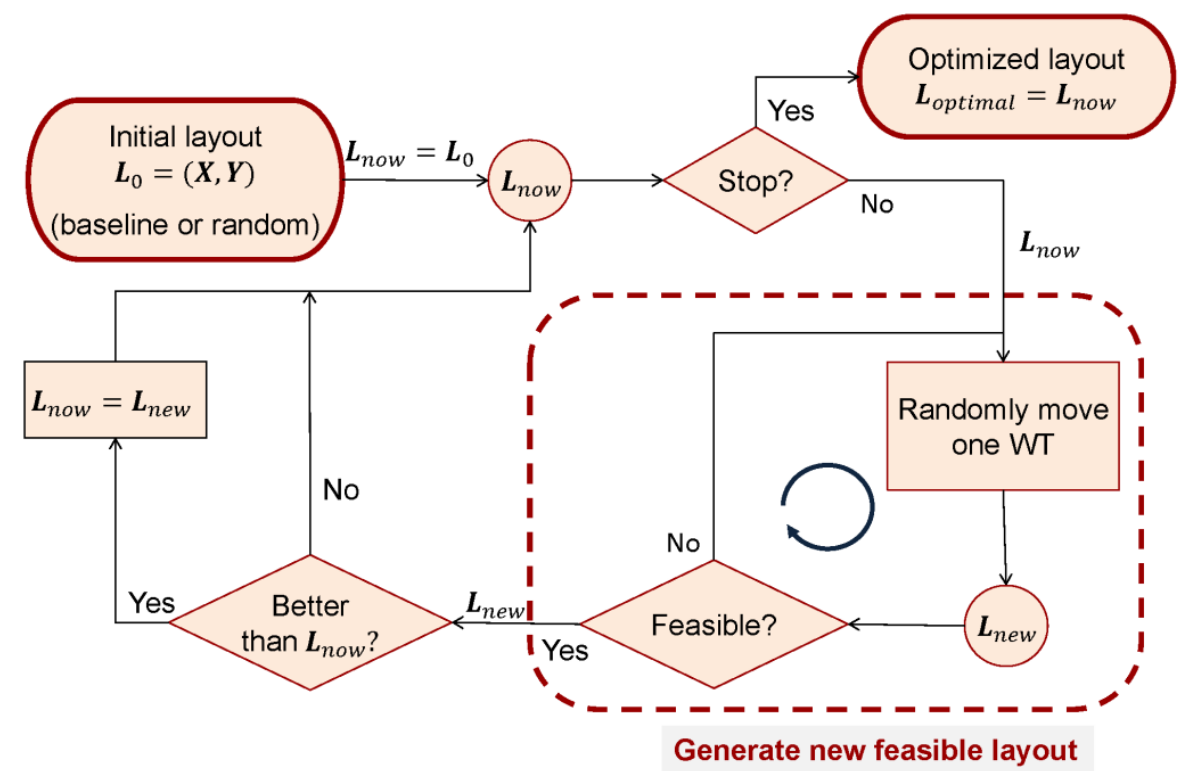

Generate new feasible layout

Figure 7. Flow chart of the random search algorithm

For each optimization problem with a different value of $\gamma$, we run the optimization algorithm with 10000 times of evaluation. The obtained results are summarized in Table 3.

Table 3. Performance summary of the original and optimized WFs with different $\gamma$

\begin{tabular}{llllll}
\hline & $P_{\text {mean }}[\mathrm{MW}]$ & VoP [-] & $R_{\text {short }}[-]$ & $R_{\text {long }}[-]$ & $I m p[-]$ \\
\hline Original & 79.61 & $2.63 \mathrm{e}-4$ & 1.000 & 4.750 & - \\
Optimal $(\gamma=0)$ & $79.71(+0.13 \%)$ & $2.13 \mathrm{e}-4(-19.12 \%)$ & $1.012(+1.19 \%)$ & $4.754(+0.09 \%)$ & $0.09 \%$ \\
Optimal $(\gamma=0.05)$ & $79.72(+0.14 \%)$ & $2.09 \mathrm{e}-4(-20.78 \%)$ & $1.013(+1.30 \%)$ & $4.754(+0.10 \%)$ & $0.11 \%$ \\
Optimal $(\gamma=0.5)$ & $79.68(+0.08 \%)$ & $1.85 \mathrm{e}-4(-29.70 \%)$ & $1.019(+1.86 \%)$ & $4.753(+0.06 \%)$ & $0.38 \%$ \\
Optimal $(\gamma=\mathbf{0 . 9 5})$ & $\mathbf{7 9 . 7 1 ( + \mathbf { 0 . 1 2 } \% )}$ & $\mathbf{1 . 8 1 e - 4}(\mathbf{- 3 1 . 1 0} \%)$ & $\mathbf{1 . 0 2 0}(+\mathbf{2 . 0 0 \% )}$ & $\mathbf{4 . 7 5 2}(+\mathbf{0 . 0 6 \%} \%$ & $\mathbf{1 . 6 1 \%}$
\end{tabular}




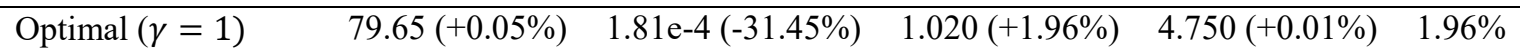

Note that in the above table $\operatorname{Imp}$ is the relative improvement of $R_{\text {overall }}$. The results in Table 3 show: the increased power production $\left(P_{\text {mean }}\right)$ and decreased variability $(V o P)$ can be achieved with a modest number of evaluations $(10000)$ for all cases; $P_{\text {mean }}$ is only slightly increased while VoP is largely decreased; the short-term robustness $\left(R_{\text {short }}\right)$ is easier to improve compared with the long-term robustness $\left(R_{\text {long }}\right)$; different values of $\gamma$ result in different trade-off between the involved WF characteristics $\left(P_{\text {mean }}, V o P, R_{\text {short }}\right.$ and $\left.R_{\text {long }}\right)$. Comparing the results for all cases, we can conclude that $\gamma=0.95$ is a good choice that achieves balanced improvements over all quantities and thus should be recommended as the default weighting parameter between the short-term and long-term robustness. Nevertheless, we should remember that the weighting parameters $(\alpha=\beta=\gamma=0.95)$ used here are recommended only as the default values and they are free to tune and should be tuned by the WF developers according to their preferences.

Figure 8 illustrates the optimization result for the case with $\gamma=0.95$. The optimized WF is shown to have a wind direction changes.

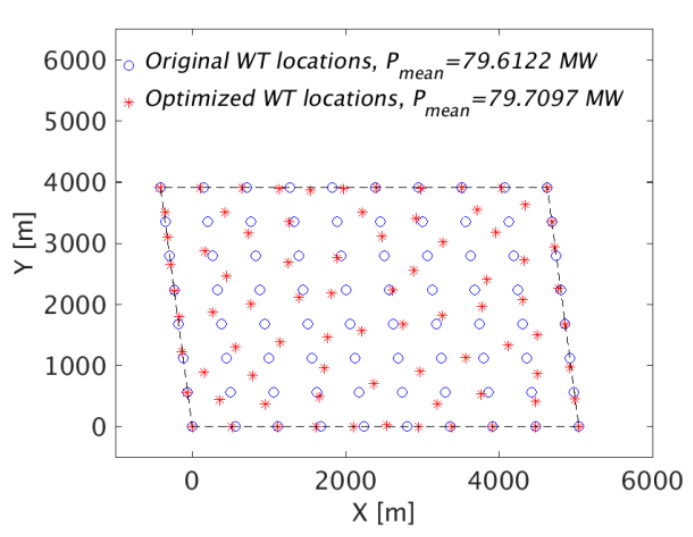

(a)

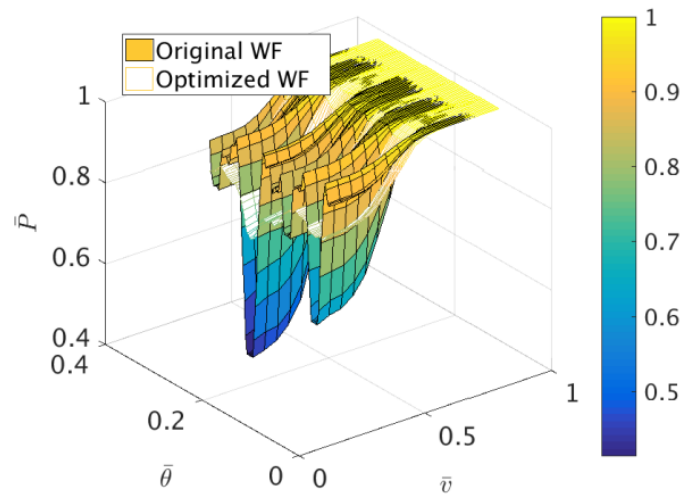

(b) 


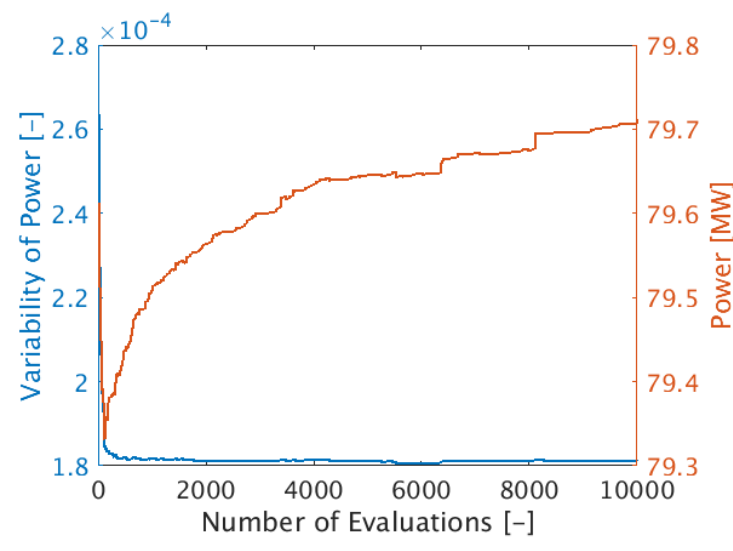

(c)

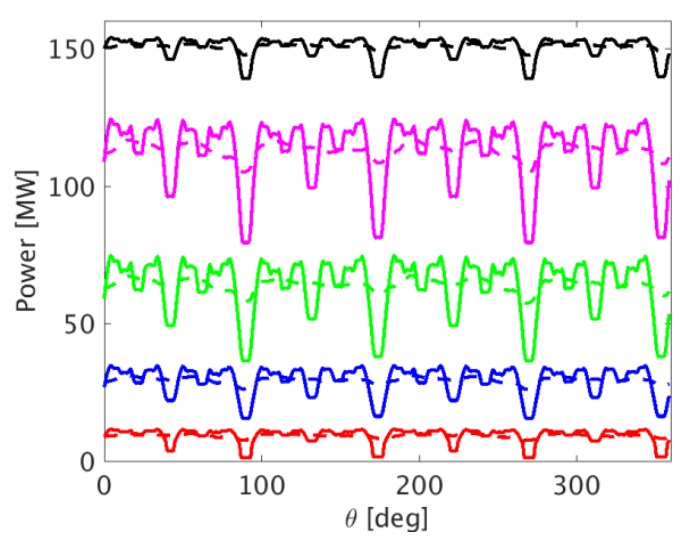

(d)

Figure 8. Layout optimization results of Horns Rev $1 \mathrm{WF}$ to maximize $R_{\text {overall }}(\gamma=0.95)$ : (a) layouts; (b) partial dimensionless power surfaces; (c) evolution histories of $V o P$ and $P_{\text {mean }}$ in the optimization process; (d) power outputs for all wind directions with different wind speeds (from bottom to top: $v=5,7,9,11,13 \mathrm{~m} / \mathrm{s}$ ) of the original (solid lines) and optimized (dash lines) WFs

When calculating the long-term robustness metric $R_{\text {long }}$ using Equation (10), we have obtained the mean and standard deviation of the series of mean power production $\left(\left[P_{\text {mean }}^{l}\right]\right)$ under long-term wind condition variations. For the case shown in Figure 8, the mean is 78.54MW for the original WF and 78.63MW for the optimized WF, while the standard deviations are both $3.482 \mathrm{MW}$ for the two WFs. It clearly shows the superiority of the optimized WF to the original WF, when facing possible long-term wind condition variations. Considering the fact that the optimized WF also has higher $P_{\text {mean }}$ and lower $V o P$, we could confidently conclude that the optimized layout is better than the original one.

\section{Conclusion}

Wind farms are operating in the constantly changing wind. Maximizing a wind farm's mean power output while minimizing its variability under both short-term and long-term wind condition variations are both important for the wind farm owner and the power system operator. The robustness quantification method proposed in this study is the first effort to capture the trade-offs between high mean power and low variability, and between shortterm and long-term performance in a single metric. The proposed overall robustness metric can be used to assess the robustness of a given WF's power production against short-term and/or long-term wind condition variations. 
The followed layout optimization study demonstrates that it can serve as a more flexible and complete objective in WF design optimization than mean power or variability alone. The results also show that we can design more robust WFs that output higher mean power and also are less sensitive to wind condition changes.

The proposed robustness metrics and associated layout optimization method have the potential to serve as a useful tool for both WF developers and power system operators. However, the full realization of this potential would require more real-life case studies and collaboration with industrial practitioners.

Currently the robustness metrics are defined solely on power production characteristics, it is also envisaged that more realistic economic models for electricity selling and related variability cost should also come into play in the future study, which will make the metrics more appealing for the potential users.

\section{Acknowledgements}

This work was supported by the international collaboration project (DSF Sagsnr. 10-094544) under Danish Research and Innovation Council for Strategic Research, and The Energy Technology Development and Demonstration Program (EUDP J.nr. 64013-0405) under Danish Energy Agency.

\section{References}

[1] The Danish Wind Industry Association, Statistics on the development of wind power in Denmark, (2016). http://windpower.org/en/knowledge/statistics/the_danish_market.html (accessed September 6, 2016).

[2] G. Boyle, Renewable electricity and the grid: the challenge of variability, Earthscan, 2007. doi:10.4324/9781849772334.

[3] W. Katzenstein, J. Apt, The cost of wind power variability, Energy Policy. 51 (2012) 233-243. doi:10.1016/j.enpol.2012.07.032.

[4] J. Kiviluoma, H. Holttinen, D. Weir, R. Scharff, L. Söder, N. Menemenlis, et al., Variability in largescale wind power generation, Wind Energy. 19 (2016) 1649-1665. doi:10.1002/we.1942.

[5] T. Boutsika, S. Santoso, Quantifying short-term wind power variability using the conditional range metric, IEEE Trans. Sustain. Energy. 3 (2012) 369-378. doi:10.1109/PES.2011.6039820. 
[6] Y. Niu, S. Santoso, Conditional Range Metric for Determining Wind Power Variability of Scarce or Noisy Data, IEEE Trans. Sustain. Energy. 6 (2015) 454-463. doi:10.1109/TSTE.2014.2379175.

[7] J. Apt, The spectrum of power from wind turbines, J. Power Sources. 169 (2007) 369-374. doi:10.1016/j.jpowsour.2007.02.077.

[8] W. Katzenstein, E. Fertig, J. Apt, The variability of interconnected wind plants, Energy Policy. 38 (2010) 4400-4410. doi:10.1016/j.enpol.2010.03.069.

[9] F. Cassola, M. Burlando, M. Antonelli, C.F. Ratto, Optimization of the Regional Spatial Distribution of Wind Power Plants to Minimize the Variability of Wind Energy Input into Power Supply Systems, J. Appl. Meteorol. Climatol. 47 (2008) 3099-3116. doi:10.1175/2008JAMC1886.1.

[10] Z. Zhang, Q. Zhou, A. Kusiak, Optimization of wind power and its variability with a computational intelligence approach, IEEE Trans. Sustain. Energy. 5 (2014) 228-236. doi:10.1109/TSTE.2013.2281354.

[11] I. Okumus, A. Dinler, Current status of wind energy forecasting and a hybrid method for hourly predictions, Energy Convers. Manag. 123 (2016) 362-371. doi:10.1016/j.enconman.2016.06.053.

[12] R. Azimi, M. Ghofrani, M. Ghayekhloo, A hybrid wind power forecasting model based on data mining and wavelets analysis, Energy Convers. Manag. 127 (2016) 208-225. doi:10.1016/j.enconman.2016.09.002.

[13] N. Kirchner-Bossi, R. García-Herrera, L. Prieto, R.M. Trigo, A long-term perspective of wind power output variability, Int. J. Climatol. 35 (2015) 2635-2646. doi:10.1002/joc.4161.

[14] S. Watson, Quantifying the variability of wind energy, Wiley Interdiscip. Rev. Energy Environ. 3 (2014) 330-342. doi:10.1002/wene.95.

[15] J. Serrano González, M. Burgos Payán, J.M.R. Santos, F. González-Longatt, A review and recent developments in the optimal wind-turbine micro-siting problem, Renew. Sustain. Energy Rev. 30 (2014) 133-144. doi:10.1016/j.rser.2013.09.027.

[16] T. Burton, N. Jenkins, D. Sharpe, E. Bossanyi, Wind Energy Handbook, 2nd ed., John Wiley \& Sons, 2011. 
[17] M. Song, K. Chen, X. Zhang, J. Wang, Optimization of wind turbine micro-siting for reducing the sensitivity of power generation to wind direction, Renew. Energy. 85 (2016) 57-65. doi:10.1016/j.renene.2015.06.033.

[18] Y. Chen, H. Li, B. He, P. Wang, K. Jin, Multi-objective genetic algorithm based innovative wind farm layout optimization method, Energy Convers. Manag. 105 (2015) 1318-1327. doi:10.1016/j.enconman.2015.09.011.

[19] J. Feng, W.Z. Shen, Solving the wind farm layout optimization problem using random search algorithm, Renew. Energy. 78 (2015) 182-192. doi:10.1016/j.renene.2015.01.005.

[20] M. De-Prada-Gil, C.G. Alías, O. Gomis-Bellmunt, A. Sumper, Maximum wind power plant generation by reducing the wake effect, Energy Convers. Manag. 101 (2015) 73-84. doi:10.1016/j.enconman.2015.05.035.

[21] J. Park, K.H. Law, Cooperative wind turbine control for maximizing wind farm power using sequential convex programming, Energy Convers. Manag. 101 (2015) 295-316. doi:10.1016/j.enconman.2015.05.031.

[22] E.C. Morgan, M. Lackner, R.M. Vogel, L.G. Baise, Probability distributions for offshore wind speeds, Energy Convers. Manag. 52 (2011) 15-26. doi:10.1016/j.enconman.2010.06.015.

[23] J. Feng, W.Z. Shen, Modelling wind for wind farm layout optimization using joint distribution of wind speed and wind direction, Energies. 8 (2015) 3075-3092. doi:10.3390/en8043075.

[24] S.J. Riley, S.D. DeGloria, R. Elliot, A Terrain Ruggedness Index that Qauntifies Topographic Heterogeneity, Intermt. J. Sci. 5 (1999) 23-27. doi:citeulike-article-id:8858430.

[25] Y.H. Wan, Long-Term Wind Power Variability, Technical Report (NREL/TP-5500-53637), National Renewable Energy Laboratory, 2012.

[26] J.A. Carta, S. Velázquez, P. Cabrera, A review of measure-correlate-predict (MCP) methods used to estimate long-term wind characteristics at a target site, Renew. Sustain. Energy Rev. 27 (2013) 362-400. doi:10.1016/j.rser.2013.07.004.

[27] J. Zhang, S. Chowdhury, A. Messac, B.M. Hodge, A hybrid measure-correlate-predict method for long- 
term wind condition assessment, Energy Convers. Manag. 87 (2014) 697-710. doi:10.1016/j.enconman.2014.07.057.

[28] T. Fang, X. Leng, X. Ma, G. Meng, $\lambda$-PDF and Gegenbauer polynomial approximation for dynamic response problems of random structures, Acta Mech. Sin. 20 (2004) 292-298.

[29] J. Feng, Y. Wang, W.-Q. Zhu, Robustness of feedback stabilization of quasi non-integrable Hamiltonian systems with parametric uncertainty, J. Vib. Control. 18 (2012) 622-631. doi:10.1177/1077546311411059. 